TAIWANESE JOURNAL OF MATHEMATICS

Vol. 16, No. 4, pp. 1489-1506, August 2012

This paper is available online at http://journal.taiwanmathsoc.org.tw

\title{
IMPLICIT AND EXPLICIT ALGORITHMS FOR MINIMUM-NORM FIXED POINTS OF PSEUDOCONTRACTIONS IN HILBERT SPACES
}

\author{
Yonghong Yao, Vittorio Colao, Giuseppe Marino and Hong-Kun $\mathrm{Xu}^{*}$
}

\begin{abstract}
We introduce implicit and explicit iterative algorithms for the construction of fixed points of pseudocontractions $T$ in Hilbert spaces. We prove that the proposed iterative algorithms converge strongly to the minimum-norm fixed point of $T$. Moreover we show that some of the existing iterative algorithms for nonexpansive mappings fail to converge when applied to pseudocontractions.
\end{abstract}

\section{INTRODUCTION}

Iterative construction of fixed points of nonlinear mappings has a long history and is still an active area of nonlinear operator theory. Let us start with the Mann iterative method which was introduced by Mann [1] in 1953 and which generates a sequence $\left(x_{n}\right)$ via the recursion:

$$
x_{n+1}=\left(1-\alpha_{n}\right) x_{n}+\alpha_{n} T x_{n}, \quad n \geq 0,
$$

where $\left(\alpha_{n}\right)$ is a sequence of real numbers in the interval $[0,1], T$ is a (nonlinear) self-mapping of a closed convex subset $C$ of a real Hilbert space $H$, and the initial guess $x_{0} \in C$ is selected arbitrarily.

Mann's method has extensively been studied in literature mainly for the class of nonexpansive mappings (recall that $T$ is nonexpansive if $\|T x-T y\| \leq\|x-y\|$ for all $x, y \in C)$. It is known [2] that if $T$ is nonexpansive with fixed points and if $\left(\alpha_{n}\right)$ satisfies the condition:

$$
\sum_{n=0}^{\infty} \alpha_{n}\left(1-\alpha_{n}\right)=\infty,
$$

Received January 21, 2011, accepted September 28, 2011.

Communicated by Chong Li.

2010 Mathematics Subject Classification: 47H05, 47H10, 47H17.

Key words and phrases: Iterative algorithm, Implicit, Explicit, Minimum-norm, Fixed point, Pseudocontraction, Nonexpansive mapping, Projection.

*Corresponding author. 
then the sequence $\left(x_{n}\right)$ generated by Mann's algorithm (1.1) converges weakly to a fixed point of $T$. (Mann's algorithm fails, in general, to converge strongly in the setting of infinite-dimensional Hilbert spaces [3].)

It is an interesting question of finding out for which class of nonlinear mappings $T$, Mann's algorithm can converge weakly (or even strongly if the space $H$ is infinitedimensional); namely, how to extend the weak convergence result of Mann's algorithm for the class of nonexpansive mappings to a wider class of nonlinear mappings. Browder and Petryshyn [4] proved the weak convergence of Mann's algorithm (1.1) for the class of strict pseudocontractions in the case of constant stepsize $\alpha_{n}=\alpha$ for all $n$ (see [5] for the general case of variable stepsize). It is however an open question whether Mann's algorithm can have weak convergence for the class of pseudocontractions in an infinite-dimensional Hilbert space. The example of Chidume and Mutangadura [6] shows that Mann's algorithm fails, in general, to converge strongly for the class of Lipschitz pseudocontractions. Therefore, Mann's algorithm is not good enough for approximating fixed points of (even if Lipschitz continuous) pseudocontractions; instead, one has to find other type of iterative algorithms. The first such an attempt was done by Ishikawa [7] who introduced the now called Ishikawa algorithm which generates a sequence $\left(x_{n}\right)$ through the recursion:

$$
\begin{aligned}
y_{n} & =\left(1-\beta_{n}\right) x_{n}+\beta_{n} T x_{n}, \quad n \geq 0, \\
x_{n+1} & =\left(1-\alpha_{n}\right) x_{n}+\alpha_{n} T y_{n},
\end{aligned}
$$

where $\left(\alpha_{n}\right)$ and $\left(\beta_{n}\right)$ are sequences of real numbers in the interval $[0,1], T$ is a (nonlinear) self-mapping of $C$, and the initial guess $x_{0} \in C$ is selected arbitrarily. (Ishikawa's algorithm (1.3) can be viewed as a double-step (or two-level) Mann's algorithm.) Ishikawa proved that his algorithm (1.3) converges in norm to a fixed point of a Lipschitz pseudocontraction $T$ if $\left(\alpha_{n}\right)$ and $\left(\beta_{n}\right)$ satisfy certain conditions and if $T$ is compact.

The purpose of this paper is twofold. Firstly, we introduce an implicit iterative algorithm which generates a sequence that converges strongly to the minimum-norm fixed point of a Lipschitz pseudocontraction $T$ in a general Hilbert space without assuming compactness of $T$. Secondly, we introduce an explicit iterative algorithm which generates a sequence that converges strongly to the minimum-norm fixed point of a strict pseudocontraction $T$ in a general Hilbert space without assuming compactness of $T$. The feature of the present paper is that, in the setting of general Hilbert spaces, our implicit (resp., explicit) iterative algorithm is not only strongly convergent, but also the limit is the minimum-norm fixed point of a Lipschitz pseudocontraction $T$ (resp., a strict pseudocontraction $T$ ) without assuming compactness of $T$, as opposed to the compactness assumption of $T$ in the existing literature (see [7] for instance), or weak convergence results only.

The paper is structured as follows. In the next section we include preliminaries- 
some notations and lemmas for the uses of the subsequent sections. In section 3 we introduce an implicit algorithm for Lipschitz pseudocontractions and prove its strong convergence to the minimum-norm fixed point of the mapping. Section 4 introduces an explicit iterative algorithm for strict pseudocontractions and proves the strong convergence of the algorithm to the minimum-norm fixed point of the mapping.

For the existing literature on iterative methods for strict pseudocontractions and pseudocontractions, the reader can consult the references $[8,9,10,11,12,13,14,15$, 16]; for nonexpansive mappings, monotone mappings and related variational inequalities, the references $[17,18,19,20,21,22]$.

We adopt the following notation:

- Fix $(S)$ stands for the set of fixed points of $S$;

- $x_{n} \rightarrow x$ stands for the weak convergence of $\left(x_{n}\right)$ to $x$;

- $x_{n} \rightarrow x$ stands for the strong convergence of $\left(x_{n}\right)$ to $x$.

\section{PReLIMINARIES}

Let $H$ be a real Hilbert space with inner product $\langle\cdot, \cdot\rangle$ and norm $\|\cdot\|$, respectively. Let $C$ be a nonempty closed convex subset of $H$. Recall the following notions for a mapping $T: C \rightarrow C$.

- $T$ is called pseudocontractive (or a pseudocontraction) if

$$
\langle T x-T y, x-y\rangle \leq\|x-y\|^{2}, \quad x, y \in C ;
$$

- $T: C \rightarrow C$ is said to be $\kappa$-strictly pseudocontractive if there exists $\kappa \in(0,1)$ such that

$$
\langle T x-T y, x-y\rangle \leq\|x-y\|^{2}-\kappa\|(I-T) x-(I-T) y\|^{2}, \quad x, y \in C ;
$$

- $T$ is nonexpansive if

$$
\|T x-T y\| \leq\|x-y\|, \quad \forall x, y \in C .
$$

It is immediately clear that nonexpansive mappings are strict pseudocontractions. It is known (and is easily seen) that

- $T$ is pseudocontractive if and only if $T$ satisfies the condition:

$$
\|T x-T y\|^{2} \leq\|x-y\|^{2}+\|(I-T) x-(I-T) y\|^{2}, \quad x, y \in C ;
$$

- $T$ is $\kappa$-strictly pseudocontractive if and only if $T$ satisfies the condition: 


$$
\|T x-T y\|^{2} \leq\|x-y\|^{2}+\kappa\|(I-T) x-(I-T) y\|^{2}, \quad x, y \in C .
$$

The (nearest point or metric) projection from $H$ onto $C$ is defined as follows: for each point $x \in H, P_{C} x$ is the unique point in $C$ with the property:

$$
\left\|x-P_{C} x\right\| \leq\|x-y\|, \quad y \in C .
$$

Note that $P_{C}$ is characterized by the inequality:

$$
P_{C} x \in C, \quad\left\langle x-P_{C} x, y-P_{C} x\right\rangle \leq 0, \quad y \in C .
$$

Consequently, $P_{C}$ is nonexpansive.

We need the following lemmas for proof of our main results.

Lemma 2.1. ([23, 24] Demiclosedness principle). Let $C$ be a nonempty closed convex subset of a real Hilbert space $H$. Let $S: C \rightarrow C$ be a non-expansive mapping with Fix $(S) \neq \emptyset$. Then $S$ is demiclosed on $C$, i.e., if $x_{n} \rightarrow x^{*} \in C$ and $x_{n}-S x_{n} \rightarrow y$, then $(I-T) x^{*}=y$.

We also need the following lemma (cf. [20]).

Lemma 2.2. Let $C$ be a nonempty closed convex subset of a real Hilbert space $H$. Assume that the mapping $F: C \rightarrow H$ is monotone and weakly continuous along segments (i.e., $F(x+t y) \rightarrow F(x)$ as $t \rightarrow 0$ ). Then the variational inequality

$$
x^{*} \in C, \quad\left\langle F x^{*}, x-x^{*}\right\rangle \geq 0, \quad x \in C .
$$

is equivalent to the dual variational inequality

$$
x^{*} \in C, \quad\left\langle F x, x-x^{*}\right\rangle \geq 0, \quad x \in C .
$$

As for strict pseudocontractions, we summarize the results contained in [4] and [5] in the following

Lemma 2.3. Assume $C$ is a closed convex subset of a Hilbert space $H$ and let $T: C \rightarrow C$ be a $\kappa$-strict pseudocontraction, then

(1) T satisfies the Lipschitz condition

$$
\|T x-T y\| \leq \frac{1+\kappa}{1-\kappa}\|x-y\|, \quad x, y \in C ;
$$

(2) the mapping $I-T$ is demiclosed at 0 (i.e. whenever $\left\{x_{n}\right\} \subset C$ is such that $x_{n} \rightarrow x$ and $(I-T) x_{n} \rightarrow 0$, then $\left.(I-T) x=0\right)$;

(3) for any constant $\gamma \in(0, \kappa)$, the map $\gamma I+(1-\gamma) T$ is nonexpansive. 
Lemma 2.4. ([18]). Let $z_{n}$ and $x_{n}$ be bounded sequences in a Banach space and $\left\{\gamma_{n}\right\}$ be a sequence in $[0,1]$ which satisfies the condition

$$
0<\liminf _{n} \leq \limsup _{n} \beta_{n}<1 .
$$

Suppose that $x_{n+1}=\left(1-\beta_{n}\right) x_{n}+\beta_{n} z_{n}$ (for all $n \geq 0$ ) satisfies the condition:

$$
\limsup _{n}\left(\left\|z_{n+1}-z_{n}\right\|-\left\|x_{n+1}-x_{n}\right\|\right) \leq 0 .
$$

Then $\lim _{n}\left\|x_{n}-z_{n}\right\|=0$.

Lemma 2.5. ([21]). Let $\left\{a_{n}\right\}_{n=0}^{\infty}$ be a sequence of nonnegative real numbers satisfying

$$
a_{n+1} \leq\left(1-\gamma_{n}\right) a_{n}+\gamma_{n} \sigma_{n}, n \geq 0,
$$

where $\left\{\gamma_{n}\right\}_{n=0}^{\infty} \subset(0,1)$ and $\left\{\sigma_{n}\right\}_{n=0}^{\infty}$ are satisfied that

(i) $\sum_{n=0}^{\infty} \gamma_{n}=\infty$;

(ii) either $\lim \sup _{n \rightarrow \infty} \sigma_{n} \leq 0$ or $\sum_{n=0}^{\infty}\left|\gamma_{n} \sigma_{n}\right|<\infty$.

Then $\left\{a_{n}\right\}_{n=0}^{\infty}$ converges to 0 .

\section{An Implicit Algorithm And Its Convergence}

In this section we introduce an implicit iterative algorithm for approximating fixed points of Lipschitz pseudocontractions $T$, and prove that our algorithm generates a strongly convergent sequence; moreover, the limit of the sequence is the minimumnorm fixed point of the mapping $T$. To define our algorithm, we assume that $C$ is a nonempty closed convex subset of a real Hilbert space $H$ such that $0 \in C, T: C \rightarrow C$ is a pseudocontraction which is also Lipschitz continuous (i.e., $\|T x-T y\| \leq L\|x-y\|$ for all $x, y \in C$ and for some constant $L$ ). Select an initial guess $x_{0} \in C$ and two real sequences $\left(\alpha_{n}\right)$ and $\left(\beta_{n}\right)$ in the unit interval $[0,1]$ such that

$$
0<\alpha_{n}+\beta_{n}<1, \quad n \geq 0 .
$$

Once the $(n-1)^{t h}$ iterate $x_{n-1}$ is defined, we define the $n^{\text {th }}$ iterate $x_{n}$ implicitly by the algorithm:

$$
x_{n}=\beta_{n} x_{n-1}+\left(1-\alpha_{n}-\beta_{n}\right) T x_{n} .
$$

Remark 3.1. We note that the algorithm (3.2) is well-defined. Indeed, for $\alpha, \beta \geq 0$ such that $0<\alpha+\beta<1$ and fixed element $u \in C$, define a mapping $U: C \rightarrow C$ by

$$
U x=\beta u+(1-\alpha-\beta) T x, \quad x \in C .
$$

Since $0 \in C, U$ is a self-mapping of $C$. Moreover, it is strongly pseudocontractive (i.e., $\langle U x-U y, x-y\rangle \leq \gamma\|x-y\|^{2}$ for $x, y \in C$, where $\gamma=1-\alpha-\beta \in(0,1)$ ). So, 
by Deimling [25], $U$ has a unique fixed point $x \in C$. This verifies that the sequence $\left\{x_{n}\right\}$ in (3.2) is well-defined.

We also note that if $\alpha_{n}=0$ for all $n$, then the algorithm (3.2) reduces to a special case of an algorithm of Xu and Ori [26] for approximating a common fixed point of a finite family of nonexpansive mappings.

In order to analyze the convergence of the algorithm (3.2), we establish the following result which plays a key role in proving the convergence Theorem 3.3.

Lemma 3.2. Let $C$ be a nonempty closed convex subset of a real Hilbert space $H$. Let $f: C \rightarrow H$ be a contraction with coefficient $\rho \in[0,1)$. Let $S: C \rightarrow C$ be a nonexpansive mapping with Fix $(S) \neq \emptyset$. For each $t \in(0,1)$, let the net $\left\{x_{t}\right\}$ be defined by

$$
x_{t}=S P_{C}\left[t f\left(x_{t}\right)+(1-t) x_{t}\right] .
$$

Then, as $t \rightarrow 0^{+}$, the net $\left\{x_{t}\right\}$ converges strongly to a point $x^{*} \in F i x(S)$ which solves the following variational inequality

$$
x^{*} \in \operatorname{Fix}(S), \quad\left\langle(I-f) x^{*}, x-x^{*}\right\rangle \geq 0, \quad x \in \operatorname{Fix}(S) .
$$

In particular, if we take $f=0$, then the net $\left\{x_{t}\right\}$ defined by

$$
x_{t}=S P_{C}\left[(1-t) x_{t}\right]
$$

converges in norm, as $t \rightarrow 0^{+}$, to the minimum-norm fixed point $x^{*} \in F i x(S)$, i.e., $x^{*}$ solves the following minimization problem

$$
x^{*} \in \operatorname{Fix}(S), \quad\left\|x^{*}\right\|=\min \{\|x\|: x \in \operatorname{Fix}(S)\} .
$$

Proof. First, we prove that the net $\left\{x_{t}\right\}$ is well-defined. For each $t \in(0,1)$, define a mapping $S_{t}: C \rightarrow C$ by

$$
S_{t} x=S P_{C}[t f(x)+(1-t) x], \quad x \in C .
$$

For $x, y \in C$, we have

$$
\begin{aligned}
\left\|S_{t} x-S_{t} y\right\| & =\left\|S P_{C}[t f(x)+(1-t) x]-S P_{C}[t f(y)+(1-t) y]\right\| \\
& \leq t\|f(x)-f(y)\|+(1-t)\|x-y\| \\
& \leq[1-(1-\rho) t]\|x-y\| .
\end{aligned}
$$

It turns out that $S_{t}$ is a contraction and hence has a unique fixed point $x_{t} \in C$. We then have

$$
x_{t}=S P_{C}\left[t f\left(x_{t}\right)+(1-t) x_{t}\right]
$$


Next we prove that $\left\{x_{t}\right\}$ is bounded. Take $u \in \operatorname{Fix}(S)$. From (3.4), we have

$$
\begin{aligned}
\left\|x_{t}-u\right\| & =\left\|S P_{C}\left[t f\left(x_{t}\right)+(1-t) x_{t}\right]-S P_{C} u\right\| \\
& \leq t\left\|f\left(x_{t}\right)-f(u)\right\|+t\|f(u)-u\|+(1-t)\left\|x_{t}-u\right\| \\
& \leq[1-(1-\rho) t]\left\|x_{t}-u\right\|+t\|f(u)-u\|,
\end{aligned}
$$

that is,

$$
\left\|x_{t}-u\right\| \leq \frac{\|f(u)-u\|}{1-\rho}
$$

Hence, $\left\{x_{t}\right\}$ is bounded. Again from (3.4), we have

$$
\begin{aligned}
\left\|x_{t}-S x_{t}\right\| & =\left\|S P_{C}\left[t f\left(x_{t}\right)+(1-t) x_{t}\right]-S P_{C} x_{t}\right\| \\
& \leq t\left\|f\left(x_{t}\right)-x_{t}\right\| \\
& \rightarrow 0 \text { as } t \rightarrow 0^{+} .
\end{aligned}
$$

Next we show that $\left\{x_{t}\right\}$ is relatively norm-compact as $t \rightarrow 0$. Let $\left\{t_{n}\right\} \subset(0,1)$ be a sequence such that $t_{n} \rightarrow 0$ as $n \rightarrow \infty$. Putting $x_{n}:=x_{t_{n}}$, we get from (3.6) that

$$
\left\|x_{n}-S x_{n}\right\| \rightarrow 0 \text {. }
$$

We compute by using (3.4)

$$
\begin{aligned}
\left\|x_{t}-u\right\|^{2}= & \left\|S P_{C}\left[t f\left(x_{t}\right)+(1-t) x_{t}\right]-S P_{C} u\right\|^{2} \\
\leq & \left\|x_{t}-u+t\left(f\left(x_{t}\right)-x_{t}\right)\right\|^{2} \\
= & \left\|x_{t}-u\right\|^{2}+2 t\left\langle f\left(x_{t}\right)-x_{t}, x_{t}-u\right\rangle+t^{2}\left\|f\left(x_{t}\right)-x_{t}\right\|^{2} \\
= & \left\|x_{t}-u\right\|^{2}+2 t\left\langle f\left(x_{t}\right)-f(u), x_{t}-u\right\rangle+2 t\left\langle f(u)-u, x_{t}-u\right\rangle \\
& +2 t\left\langle u-x_{t}, x_{t}-u\right\rangle+t^{2}\left\|f\left(x_{t}\right)-x_{t}\right\|^{2} \\
\leq & {[1-2(1-\rho) t]\left\|x_{t}-u\right\|^{2}+2 t\left\langle f(u)-u, x_{t}-u\right\rangle+t^{2}\left\|f\left(x_{t}\right)-x_{t}\right\|^{2} . }
\end{aligned}
$$

It follows that

$$
\left\|x_{t}-u\right\|^{2} \leq \frac{1}{1-\rho}\left\langle f(u)-u, x_{t}-u\right\rangle+t M
$$

where $M>0$ is a constant such that

$$
M \geq \frac{1}{2(1-\rho)} \sup \left\{\left\|f\left(x_{t}\right)-x_{t}\right\|^{2}: t \in(0,1)\right\} .
$$


Setting $t=t_{n}$ in (3.8) yields

$$
\left\|x_{n}-u\right\|^{2} \leq \frac{1}{1-\rho}\left\langle f(u)-u, x_{n}-u\right\rangle+t_{n} M, \quad u \in \operatorname{Fix}(S) .
$$

Since $\left\{x_{n}\right\}$ is bounded, we may assume, without loss of generality, that $x_{n} \rightarrow x^{*} \in C$. Then, by (3.7) and Lemma 2.1, we get $x^{*} \in F i x(S)$. Consequently, we can substitute $x^{*}$ for $u$ in (3.9) to get

$$
\left\|x_{n}-x^{*}\right\|^{2} \leq \frac{1}{1-\rho}\left\langle f\left(x^{*}\right)-x^{*}, x_{n}-x^{*}\right\rangle+t_{n} M \rightarrow 0
$$

to conclude that $\left\{x_{n}\right\}$ actually converges to $x^{*}$ strongly. This has proved the relative norm-compactness of the net $\left\{x_{t}\right\}$ as $t \rightarrow 0^{+}$.

Now we take the limit as $n \rightarrow \infty$ in (3.9) to get

$$
\left\|x^{*}-u\right\|^{2} \leq \frac{1}{1-\rho}\left\langle f(u)-u, x^{*}-u\right\rangle, \quad u \in \operatorname{Fix}(S) .
$$

In particular, $x^{*}$ solves the following variational inequality

$$
x^{*} \in F i x(S), \quad\left\langle(I-f) u, u-x^{*}\right\rangle \geq 0, \quad u \in \operatorname{Fix}(S),
$$

or the equivalent variational inequality (see Lemma 2.2)

$$
x^{*} \in \operatorname{Fix}(S),\left\langle(I-f) x^{*}, u-x^{*}\right\rangle \geq 0, \quad u \in \operatorname{Fix}(S) .
$$

Therefore, $x^{*}=\left(P_{\text {Fix }(S)} f\right) x^{*}$. That is, $x^{*}$ is the unique fixed point in $\operatorname{Fix}(S)$ of the contraction $P_{\text {Fix }(S)} f$. Clearly this is sufficient to conclude that the entire net $\left\{x_{t}\right\}$ converges in norm to $x^{*}$ as $t \rightarrow 0^{+}$.

Finally, if we take $f=0$, then variational inequality (3.10) is reduced to

$$
0 \leq\left\langle x^{*}, u-x^{*}\right\rangle, \quad u \in \operatorname{Fix}(S) .
$$

Equivalently,

$$
\left\|x^{*}\right\|^{2} \leq\left\langle x^{*}, u\right\rangle, \quad u \in \operatorname{Fix}(S) .
$$

This clearly implies that

$$
\left\|x^{*}\right\| \leq\|u\|, \quad u \in \operatorname{Fix}(S) .
$$

Therefore, $x^{*}$ is a minimum-norm fixed point of $S$. This completes the proof.

We are now in a position to prove the strong convergence of the implicit algorithm (3.2) to the minimum-norm fixed point of the pseudocontractive mapping $T$. 
Theorem 3.3. Let $C$ be a nonempty closed convex subset of a real Hilbert space $H$ such that $0 \in C$. Let $T: C \rightarrow C$ be a continuous pseudocontraction with Fix $(T) \neq \emptyset$. In addition to (3.1), assume

(i) $\lim _{n \rightarrow \infty} \alpha_{n}=\lim _{n \rightarrow \infty} \beta_{n}=0$;

(ii) $\sum_{n=0}^{\infty} \alpha_{n}=\infty$.

Then the sequence $\left\{x_{n}\right\}$ generated by the implicit algorithm (3.2) converges strongly to the minimum-norm fixed point of $T$.

Proof. First, we show that the sequence $\left\{x_{n}\right\}$ is bounded. Taking $p \in \operatorname{Fix}(T)$, we get from (3.2) and (2.1)

$$
\begin{aligned}
\left\|x_{n}-p\right\|^{2} & =\left\langle\beta_{n} x_{n-1}+\left(1-\alpha_{n}-\beta_{n}\right) T x_{n}-p, x_{n}-p\right\rangle \\
& =\left\langle\beta_{n}\left(x_{n-1}-p\right)-\alpha_{n} p, x_{n}-p\right\rangle+\left(1-\alpha_{n}-\beta_{n}\right)\left\langle T x_{n}-p, x_{n}-p\right\rangle \\
& \leq\left(\beta_{n}\left\|x_{n-1}-p\right\|+\alpha_{n}\|p\|\right)\left\|x_{n}-p\right\|+\left(1-\alpha_{n}-\beta_{n}\right)\left\|x_{n}-p\right\|^{2} .
\end{aligned}
$$

It turns that

$$
\begin{aligned}
\left\|x_{n}-p\right\| & \leq \frac{\beta_{n}}{\alpha_{n}+\beta_{n}}\left\|x_{n-1}-p\right\|+\frac{\alpha_{n}}{\alpha_{n}+\beta_{n}}\|p\| \\
& \leq \max \left\{\left\|x_{n-1}-p\right\|,\|p\|\right\} .
\end{aligned}
$$

By induction, we obtain

$$
\left\|x_{n}-p\right\| \leq \max \left\{\left\|x_{0}-p\right\|,\|p\|\right\}, \quad n \geq 0 .
$$

Consequently, $\left\{x_{n}\right\}$ is bounded.

Set $S=(2 I-T)^{-1}$ (i.e., $S$ is a resolvent of the monotone mapping $I-T$ ). It is known that $S$ is nonexpansive and $\operatorname{Fix}(S)=F i x(T)$ (cf. [27, Theorem 6]). Since

$$
\begin{aligned}
\left\|x_{n}-T x_{n}\right\| & =\left\|\beta_{n} x_{n-1}+\left(1-\alpha_{n}-\beta_{n}\right) T x_{n}-T x_{n}\right\| \\
& \leq \beta_{n}\left\|x_{n-1}-T x_{n}\right\|+\alpha_{n}\left\|T x_{n}\right\| \leq\left(\alpha_{n}+\beta_{n}\right) M_{1},
\end{aligned}
$$

for a constant $M_{1}$ such that $M_{1} \geq\left\|x_{n}\right\|+\left\|T x_{m}\right\|$ for all $m, n$, we have

$$
\begin{aligned}
\left\|x_{n}-S x_{n}\right\| & =\left\|S S^{-1} x_{n}-S x_{n}\right\| \leq\left\|S^{-1} x_{n}-x_{n}\right\| \\
& =\left\|(2 I-T) x_{n}-x_{n}\right\|=\left\|x_{n}-T x_{n}\right\| \\
& \leq\left(\alpha_{n}+\beta_{n}\right) M_{1} \rightarrow 0 .
\end{aligned}
$$

Let now $z_{t}$ be the unique fixed point of the contraction

$$
S_{t} z:=S P_{C}[(1-t) z]=S[(1-t) z], \quad z \in C, t \in(0,1) .
$$


(Note that since $0 \in C,(1-t) z \in C$ for $z \in C$.) By Lemma 3.2, $\left(z_{t}\right)$ converges in norm, as $t \rightarrow 0^{+}$, to the minimum-norm fixed point $x^{*}$ of $S$ (and of $T$ for $\operatorname{Fix}(S)=$ Fix $(T)$ ). We next claim that

$$
\limsup _{n \rightarrow \infty}\left\langle x^{*}, x^{*}-x_{n}\right\rangle \leq 0 .
$$

To see this, we use the equation $z_{t}=S\left[(1-t) z_{t}\right]$ to derive that

$$
\begin{aligned}
\left\|z_{t}-x_{n}\right\|^{2} & =\left\|S\left[(1-t) z_{t}\right]-S x_{n}+S x_{n}-x_{n}\right\|^{2} \\
& \leq\left\|S\left[(1-t) z_{t}\right]-S x_{n}\right\|^{2}+2\left\langle S x_{n}-x_{n}, z_{t}-x_{n}\right\rangle \\
& \leq\left\|z_{t}-x_{n}-t z_{t}\right\|^{2}+2\left\|z_{t}-x_{n}\right\|\left\|S x_{n}-x_{n}\right\| \\
& =\left\|z_{t}-x_{n}\right\|^{2}-2 t\left\langle z_{t}, z_{t}-x_{n}\right\rangle+t^{2}\left\|z_{t}\right\|^{2}+2\left\|z_{t}-x_{n}\right\|\left\|S x_{n}-x_{n}\right\| \\
& \leq\left\|z_{t}-x_{n}\right\|^{2}-2 t\left\langle z_{t}, z_{t}-x_{n}\right\rangle+M_{2}\left(t^{2}+\left\|S x_{n}-x_{n}\right\|\right),
\end{aligned}
$$

where $M_{2}$ is a constant such that $M_{2} \geq \sup \left\{\left\|z_{t}\right\|^{2}+2\left\|z_{t}-x_{n}\right\|: t \in(0,1), n \geq 0\right\}$. It turns out that

$$
\left\langle z_{t}, z_{t}-x_{n}\right\rangle \leq \frac{M_{2} t}{2}+\frac{M_{2}}{2 t}\left\|S x_{n}-x_{n}\right\| .
$$

Letting $n \rightarrow \infty$ in (3.14) and noting (3.12), we have

$$
\limsup _{n \rightarrow \infty}\left\langle z_{t}, z_{t}-x_{n}\right\rangle \leq \frac{M_{2} t}{2}
$$

Therefore,

$$
\limsup _{t \rightarrow 0^{+}} \limsup _{n \rightarrow \infty}\left\langle z_{t}, z_{t}-x_{n}\right\rangle \leq 0 .
$$

This, together with the strong convergence of $\left(z_{t}\right)$ to $x^{*}$, implies (3.13).

Finally, we prove that $x_{n} \rightarrow x^{*}$. From (3.2) and (2.1), we have

$$
\begin{aligned}
& \left\|x_{n}-x^{*}\right\|^{2} \\
= & \left\langle\beta_{n} x_{n-1}+\left(1-\alpha_{n}-\beta_{n}\right) T x_{n}-x^{*}, x_{n}-x^{*}\right\rangle \\
= & \beta_{n}\left\langle x_{n-1}-x^{*}, x_{n}-x^{*}\right\rangle+\left(1-\alpha_{n}-\beta_{n}\right)\left\langle T x_{n}-x^{*}, x_{n}-x^{*}\right\rangle-\alpha_{n}\left\langle x^{*}, x_{n}-x^{*}\right\rangle \\
\leq & \beta_{n}\left\|x_{n-1}-x^{*}\right\|\left\|x_{n}-x^{*}\right\|+\left(1-\alpha_{n}-\beta_{n}\right)\left\|x_{n}-x^{*}\right\|^{2}+\alpha_{n}\left\langle x^{*}, x^{*}-x_{n}\right\rangle \\
\leq & \frac{\beta_{n}}{2}\left(\left\|x_{n-1}-x^{*}\right\|^{2}+\left\|x_{n}-x^{*}\right\|^{2}\right)+\left(1-\alpha_{n}-\beta_{n}\right)\left\|x_{n}-x^{*}\right\|^{2} \\
& +\alpha_{n}\left\langle x^{*}, x^{*}-x_{n}\right\rangle .
\end{aligned}
$$

It turns out that 


$$
\begin{aligned}
\left\|x_{n}-x^{*}\right\|^{2} & \leq \frac{\beta_{n}}{2 \alpha_{n}+\beta_{n}}\left\|x_{n-1}-x^{*}\right\|^{2}+\frac{2 \alpha_{n}}{2 \alpha_{n}+\beta_{n}}\left\langle x^{*}, x^{*}-x_{n}\right\rangle \\
& =\left(1-\frac{2 \alpha_{n}}{2 \alpha_{n}+\beta_{n}}\right)\left\|x_{n-1}-x^{*}\right\|^{2}+\frac{2 \alpha_{n}}{2 \alpha_{n}+\beta_{n}}\left\langle x^{*}, x^{*}-x_{n}\right\rangle \\
& =\left(1-\gamma_{n}\right)\left\|x_{n-1}-x^{*}\right\|^{2}+\gamma_{n} \sigma_{n}
\end{aligned}
$$

where

$$
\gamma_{n}=\frac{2 \alpha_{n}}{2 \alpha_{n}+\beta_{n}} \quad \text { and } \quad \sigma_{n}=\left\langle x^{*}, x^{*}-x_{n}\right\rangle .
$$

Since $\lim _{n \rightarrow \infty} \alpha_{n}=\lim _{n \rightarrow \infty} \beta_{n}=0$, we may assume that $2 \alpha_{n}+\beta_{n} \leq 1$ for $n$. Hence, $\gamma_{n} \geq 2 \alpha_{n}$ and $\sum_{n=0}^{\infty} \gamma_{n}=\infty$ due to condition (ii) of the theorem. We also have by (3.13) that $\lim \sup _{n \rightarrow \infty} \sigma_{n} \leq 0$. Therefore, Lemma 2.5 is applicable to (3.15) and we conclude that $x_{n} \rightarrow x^{*}$ as $n \rightarrow \infty$.

Remark 3.4. In Theorem 3.3 we assumed that $0 \in C$. We do not know if this assumption can be removed. The difficulty without assuming $0 \in C$ lies in that the contraction mapping $U$ defined in (3.3) may fail to be a self-mapping of $C$ and thus $U$ may be fixed point free. As a result, the algorithm (3.2) may not be well-defined. It is of interest to adapt the algorithm (3.2) to suit for the general case (i.e., without assuming $0 \in C$ ) of find the minimum-norm fixed point of a Lipschitz pseudocontraction.

Remark 3.5. For a nonexpansive map $S$, the following explicit algorithm

$$
\left\{\begin{array}{l}
y_{n}=P_{C}\left(1-\alpha_{n}\right) x_{n} \\
x_{n+1}=\left(1-\delta_{n}\right) x_{n}+\delta_{n} S y_{n}
\end{array}\right.
$$

is introduced in [19] to approximate the minimum-norm fixed point of $S$, where $\left(\alpha_{n}\right)$ and $\left(\delta_{n}\right)$ are real sequences in the interval $[0,1]$ satisfying certain conditions.

On the other hand, we may naturally consider an explicit version of the implicit algorithm (3.2) which generates a $\left\{w_{n}\right\}$ by the iterative procedure:

$$
w_{n+1}=\beta_{n} w_{n}+\left(1-\alpha_{n}-\beta_{n}\right) T w_{n} .
$$

A natural question that arises is whether the sequence $\left(x_{n}\right)$ generated by either of the algorithms (3.16) and (3.17) can converge for Lipschitz pseudo-contractions $T$. we will provide an example to answer this question in the negative.

Example 3.6. (Chidume and Mutangadura [6].) Let $H=\mathbb{R}^{2}$ and $B:=\overline{B(0,1)}$. Fix $x_{0} \in B \backslash\{0\}$ and $\left\{\alpha_{n}\right\},\left\{\beta_{n}\right\},\left\{\delta_{n}\right\} \subset(0,1)$ such that

(1) $\lim _{n} \alpha_{n}=0$

(2) $\lim _{n} \beta_{n}=0$ and

(3) $\liminf _{n} \delta_{n}>0$. 
Then there exists a Lipschitz pseudocontraction $T: B \rightarrow B$ such that the sequences

$$
\left\{\begin{array}{l}
y_{n}=\left(1-\alpha_{n}\right) x_{n} \\
x_{n+1}=\left(1-\delta_{n}\right) x_{n}+\delta_{n} T y_{n}
\end{array}\right.
$$

and

$$
w_{n+1}=\beta_{n} w_{n}+\left(1-\alpha_{n}-\beta_{n}\right) T w_{n}
$$

do not converge to the fixed point of $T$ for any nonzero initial choice of $x_{0}$ and $w_{0}$.

Proof. Let $B_{1 / 2}=\overline{B(0,1 / 2)}$ and for $x \in \mathbb{R}^{2}$, denote by $x^{\perp}$ the unique element such that $\left\langle x, x^{\perp}\right\rangle=0$. Following [6], we define $T: B \rightarrow B$ by

$$
\begin{cases}x+x^{\perp}, & \text { if } x \in B_{1 / 2}, \\ \frac{x}{\|x\|}-x+x^{\perp}, & \text { if } x \in B \backslash B_{1 / 2} .\end{cases}
$$

It is proved [6] that $T$ is a Lipschitz pseudocontraction and $x=0$ is the unique fixed point of $T$.

Since $\lim _{n} \alpha_{n}=0$ and $\liminf _{n} \delta_{n}>0$, we may assume that

(1) $r_{n}:=\delta_{n}^{2}+\alpha_{n}\left(\alpha_{n} \delta_{n}^{2}-\delta_{n}^{2}-2 \delta_{n}\right)>0$ and

(2) $s_{n}:=\delta_{n}^{2}+\alpha_{n}\left(2 \delta_{n}-2 \alpha_{n}+2 \alpha_{n} \delta_{n}^{2}\right)>0$

for all $n$. If, for a fixed $n \in \mathbb{N}$, we assume that $\left(1-\alpha_{n}\right) x \in B_{1 / 2} \backslash\{0\}$, then

$$
\begin{aligned}
& \left\|\left(1-\delta_{n}\right) x+\delta_{n} T\left(1-\alpha_{n}\right) x\right\|^{2} \\
= & \left\|\left(1-\delta_{n}\right) x+\delta_{n}\left(1-\alpha_{n}\right) x+\delta_{n}\left(1-\alpha_{n}\right) x^{\perp}\right\|^{2} \\
= & \left\|\left(1-\alpha_{n} \delta_{n}\right) x+\left(\delta_{n}-\alpha_{n} \delta_{n}\right) x^{\perp}\right\|^{2} \\
= & \left(\left(1-\alpha_{n} \delta_{n}\right)^{2}+\left(\delta_{n}-\alpha_{n} \delta_{n}\right)^{2}\right)\|x\|^{2} \\
= & \left(1+r_{n}\right)\|x\|^{2} \\
> & \|x\|^{2} .
\end{aligned}
$$

On the other hand, if $\left(1-\alpha_{n}\right) x \in B \backslash B_{1 / 2}$ we have

$$
\begin{aligned}
& \left\|\left(1-\delta_{n}\right) x_{n}+\delta_{n} T\left(1-\alpha_{n}\right) x\right\|^{2} \\
= & \left\|\left(1-\delta_{n}\right) x+\delta_{n} \frac{x}{\|x\|}-\delta_{n}\left(1-\alpha_{n}\right) x+\delta_{n}\left(1-\alpha_{n}\right) x^{\perp}\right\|^{2} \\
= & \left\|\left(\frac{\delta_{n}}{\|x\|}+1-2 \delta_{n}+\alpha_{n} \delta_{n}\right) x+\delta_{n}\left(1-\alpha_{n}\right) x^{\perp}\right\|^{2} \\
= & \left(\left(\frac{\delta_{n}}{\|x\|}+1-2 \delta_{n}+\alpha_{n} \delta_{n}\right)^{2}+\delta_{n}^{2}\left(1-\alpha_{n}\right)^{2}\right)\|x\|^{2} \\
\geq & \left(1+2 \alpha_{n} \delta_{n}+2 \alpha_{n}^{2} \delta_{n}^{2}+\delta_{n}^{2}-2 \alpha_{n} \delta_{n}^{2}\right)\|x\|^{2} \\
= & \left(1+s_{n}\right)\|x\|^{2} \\
> & \|x\|^{2} .
\end{aligned}
$$


We thus get from (3.20) and (3.21) that

$$
\left\|x_{n+1}\right\|>\left\|x_{n}\right\| .
$$

Hence the sequence $\left(x_{n}\right)$ generated by the algorithm (3.18) does not converge to the unique fixed point 0 of $T$.

We next turn to the sequence $\left(w_{n}\right)$ generated by the algorithm (3.19). Suppose on the contrary that $\left(w_{n}\right)$ converges to 0 . Then by continuity of $T$ and by conditions over $\left\{\alpha_{n}\right\}$ and $\left\{\beta_{n}\right\}$, we can assume that, for some $\bar{n}$ large enough and for any $n \geq \bar{n}$, $w_{n} \in B_{1 / 2} \backslash\{0\}$ and

$$
\left(1-\alpha_{n}\right)^{2}+\left(1-\beta_{n}-\alpha_{n}\right)^{2}>1 .
$$

It follows that, for $n \geq \bar{n}$,

$$
\begin{aligned}
\left\|w_{n+1}\right\|^{2} & =\left\|\beta_{n} w_{n}+\left(1-\alpha_{n}-\beta_{n}\right) T w_{n}\right\|^{2} \\
& =\left\|\beta_{N} w_{n}+\left(1-\alpha_{n}-\beta_{n}\right) w_{n}+\left(1-\alpha_{n}-\beta_{n}\right) w_{n}^{\perp}\right\|^{2} \\
& =\left(1-\alpha_{n}\right)^{2}\left\|w_{n}\right\|^{2}+\left(1-\alpha_{n}-\beta_{n}\right)^{2}\left\|w_{n}^{\perp}\right\|^{2} \\
& =\left(\left(1-\alpha_{n}\right)^{2}+\left(1-\alpha_{n}-\beta_{n}\right)^{2}\right)\left\|w_{n}\right\|^{2} \\
& >\left\|w_{n}\right\|^{2} .
\end{aligned}
$$

Consequently, the sequence $\left\{w_{n}\right\}$ cannot converge to 0 and we have reached a contradiction.

\section{An Explicit Algorithm and Its Convergence}

Example 3.6 shows that the explicit algorithm (3.18) fails, in general, to converge to a fixed point of a Lipschitz pseudocontraction. The purpose of this section is to prove that a slight modification of this algorithm does indeed converge for strict pseudocontractions (without assuming $0 \in C$ ), and the limit is moreover the minimumnorm fixed point of the mapping.

Theorem 4.1. Let $C$ be a nonempty closed convex subset of a real Hilbert space $H$. Let $T: C \rightarrow C$ be a $\kappa$-strict pseudocontraction with Fix $(T) \neq \emptyset$. Let $\left\{\alpha_{n}\right\},\left\{\delta_{n}\right\} \subset$ $(0,1)$ and $\left(\gamma_{n}\right) \subset(0, \kappa)$ be real sequences such that

(1) $\lim _{n} \alpha_{n}=0$,

(2) $\sum_{n} \alpha_{n}=\infty$ and

(3) $0<\liminf _{n} \delta_{n} \leq \lim \sup _{n} \delta_{n}<1$.

For a given initial guess $x_{0} \in C$, let $\left(x_{n}\right)$ be generated by the explicit algorithm

$$
\left\{\begin{array}{l}
y_{n}=P_{C}\left[\left(1-\alpha_{n}\right) x_{n}\right] \\
x_{n+1}=\left(1-\delta_{n}\right) x_{n}+\delta_{n} \gamma_{n} y_{n}+\delta_{n}\left(1-\gamma_{n}\right) T y_{n} .
\end{array}\right.
$$

Then $\left(x_{n}\right)$ converges strongly to the minimum-norm fixed point of $T$. 
Proof. First observe by Lemma 2.3 that, for each fixed $n \in \mathbb{N}$, the mapping

$$
T_{n}:=\gamma_{n} I+\left(1-\gamma_{n}\right) T
$$

is nonexpansive and $\operatorname{Fix}(T)=\operatorname{Fix}\left(T_{n}\right)$. Moreover, we may rewrite (4.1) as

$$
\left\{\begin{array}{l}
y_{n}=P_{C}\left[\left(1-\alpha_{n}\right) x_{n}\right] \\
x_{n+1}=\left(1-\delta_{n}\right) x_{n}+\delta_{n} T_{n} y_{n} .
\end{array}\right.
$$

To discuss the convergence analysis for the sequence $\left(x_{n}\right)$, we first prove the boundedness of $\left(x_{n}\right)$, towards which we take $q \in F i x(T)$ and infer that

$$
\begin{aligned}
\left\|x_{n+1}-q\right\| & =\left\|\left(1-\delta_{n}\right) x_{n}+\delta_{n} T_{n} y_{n}-q\right\| \\
& \leq\left(1-\delta_{n}\right)\left\|x_{n}-q\right\|+\delta_{n}\left\|T_{n} y_{n}-T_{n} q\right\| \\
& \leq\left(1-\delta_{n}\right)\left\|x_{n}-q\right\|+\delta_{n}\left\|\left(1-\alpha_{n}\right) x_{n}-q\right\| \\
& \leq\left(\left(1-\delta_{n}\right)+\beta_{n}\left(1-\alpha_{n}\right)\right)\left\|x_{n}-q\right\|+\alpha_{n} \delta_{n}\|q\| \\
& =\left(1-\delta_{n} \alpha_{n}\right)\left\|x_{n}-q\right\|+\alpha_{n} \delta_{n}\|q\| \\
& \leq \max \left\{\left\|x_{n}-q\right\|,\|q\|\right\} .
\end{aligned}
$$

By induction, we get

$$
\left\|x_{n}-q\right\| \leq \max \left\{\left\|x_{0}-q\right\|,\|q\|\right\}, \quad n \geq 0
$$

In particular, $\left(x_{n}\right)$ is bounded. Next we show that

$$
\lim _{n}\left\|x_{n}-T x_{n}\right\|=0 .
$$

To see this, we set $z_{n}=T_{n} y_{n}$. From the nonexpansivity of $T_{n}$, it follows that

$$
\begin{aligned}
\left\|z_{n+1}-z_{n}\right\| & \leqslant P_{C}\left(1-\alpha_{n+1}\right) x_{n+1}-P_{C}\left(1-\alpha_{n}\right) x_{n} \| \\
& \leq \alpha_{n+1}\left\|x_{n+1}\right\|+\alpha_{n}\left\|x_{n}\right\|+\left\|x_{n+1}-x_{n}\right\|
\end{aligned}
$$

so that

$$
\limsup _{n}\left(\left\|z_{n+1}-z_{n}\right\|-\left\|x_{n+1}-x_{n}\right\|\right)=0
$$

We can therefore apply Lemma 2.4 to obtain

$$
\lim _{n}\left\|x_{n}-z_{n}\right\|=0 \text {. }
$$

By (4.2), we deduce that

$$
\begin{aligned}
\left\|x_{n}-T y_{n}\right\| & =\frac{1}{1-\gamma_{n}}\left\|\left(1-\gamma_{n}\right) x_{n}-\left(1-\gamma_{n}\right) T y_{n}\right\| \\
& =\frac{1}{1-\gamma_{n}}\left\|\gamma_{n}\left(y_{n}-x_{n}\right)+x_{n}-\gamma_{n} y_{n}-\left(1-\gamma_{n}\right) T y_{n}\right\| \\
& \leq \frac{1}{1-\gamma_{n}}\left(\gamma_{n}\left\|P_{C}\left(1-\alpha_{n}\right) x_{n}-x_{n}\right\|+\left\|x_{n}-z_{n}\right\|\right) \\
& \leq \frac{1}{1-\gamma_{n}}\left(\gamma_{n} \alpha_{n}\left\|x_{n}\right\|+\left\|x_{n}-z_{n}\right\|\right)
\end{aligned}
$$


Passing to the limit as $n \rightarrow \infty$ in the last relation and using (4.4), we get

$$
\lim _{n}\left\|x_{n}-T y_{n}\right\|=0
$$

Again by (4.2), we have

$$
\lim _{n}\left\|x_{n+1}-x_{n}\right\|=\lim _{n} \delta_{n}\left\|z_{n}-x_{n}\right\|=0
$$

Now since $T$ is Lipschitz with constant $L=(1+\kappa) /(1-\kappa)$ by Lemma 2.3 , it follows that

$$
\begin{aligned}
\left\|x_{n}-T x_{n}\right\| \leq & \left\|x_{n}-x_{n+1}\right\|+\left\|x_{n+1}-T x_{n}\right\| \\
\leq & \left\|x_{n}-x_{n+1}\right\|+\left(1-\delta_{n}\right)\left\|x_{n}-T x_{n}\right\|+\delta_{n} \gamma_{n}\left\|T x_{n}-y_{n}\right\| \\
& +\delta_{n}\left(1-\gamma_{n}\right)\left\|T x_{n}-T y_{n}\right\| \\
\leq & \left\|x_{n}-x_{n+1}\right\|+\left(1-\delta_{n}\right)\left\|x_{n}-T x_{n}\right\|+\delta_{n} \gamma_{n}\left(\left\|T x_{n}-T y_{n}\right\|\right. \\
& \left.+\left\|T y_{n}-x_{n}\right\|+\left\|x_{n}-y_{n}\right\|\right)+L \delta_{n}\left(1-\gamma_{n}\right)\left\|x_{n}-y_{n}\right\| \\
\leq & \left\|x_{n}-x_{n+1}\right\|+\left(1-\delta_{n}\right)\left\|x_{n}-T x_{n}\right\|+\delta_{n} \gamma_{n}\left\|T y_{n}-x_{n}\right\| \\
& +\delta_{n}\left(\gamma_{n}+L\right)\left\|x_{n}-y_{n}\right\| .
\end{aligned}
$$

But, since

$$
\left\|x_{n}-y_{n}\right\|=\left\|x_{n}-P_{C}\left[\left(1-\alpha_{n}\right) x_{n}\right]\right\| \leq \alpha_{n}\left\|x_{n}\right\|
$$

we get from (4.6) that

$$
\left\|x_{n}-T x_{n}\right\| \leq \frac{\left\|x_{n}-x_{n+1}\right\|}{\delta_{n}}+\gamma_{n}\left\|T y_{n}-x_{n}\right\|+\alpha_{n}\left(\gamma_{n}+L\right)\left\|x_{n}\right\| \rightarrow 0
$$

and (4.3) is proven.

Let $x^{*}$ be the minimum-norm fixed point of $T$ (i.e., $x^{*}=\arg \min _{x \in \operatorname{Fix}(T)}\|x\|$ ) and let $\left(x_{n_{i}}\right)$ be a subsequence of $\left(x_{n}\right)$ such that

$$
\limsup _{n}\left\langle x^{*}, x^{*}-x_{n}\right\rangle=\lim _{i}\left\langle x^{*}, x^{*}-x_{n_{i}}\right\rangle .
$$

Due to boundedness of $\left(x_{n}\right)$, we may assume that $\left(x_{n_{i}}\right)$ weakly converges to a point $u$. By (4.3) and the demiclosedness of $I-T$, we have $u \in F i x(T)$. It follows from (4.7) that

$$
\limsup _{n}\left\langle x^{*}, x^{*}-x_{n}\right\rangle=\left\langle x^{*}, x^{*}-u\right\rangle \leq 0
$$

by the characterization of projections (2.5). 
We can now prove the strong convergence $\left(x_{n}\right)$ to $x^{*}$. Setting $a_{n}=\left\|x_{n}-x^{*}\right\|^{2}$, we get (noticing that $T_{n}$ is nonexpansive and $T_{n} x^{*}=x^{*}$ for all $n$ )

$$
\begin{aligned}
& a_{n+1} \\
= & \left\|\left(1-\delta_{n}\right)\left(x_{n}-x^{*}\right)+\delta_{n}\left(T_{n} y_{n}-x^{*}\right)\right\|^{2} \\
\leq & \left(1-\delta_{n}\right)\left\|x_{n}-x^{*}\right\|^{2}+\delta_{n}\left\|y_{n}-x^{*}\right\|^{2} \\
= & \left(1-\delta_{n}\right)\left\|x_{n}-x^{*}\right\|^{2}+\delta_{n}\left\|P_{C}\left[\left(1-\alpha_{n}\right) x_{n}\right]-x^{*}\right\|^{2} \\
\leq & \left(1-\delta_{n}\right) a_{n}+\delta_{n}\left\|\left(1-\alpha_{n} x_{n}\right)-x^{*}\right\|^{2} \\
\leq & \left(1-\delta_{n}\right) a_{n}+\delta_{n}\left(1-\alpha_{n}\right) a_{n}+\delta_{n} \alpha_{n}^{2}\left\|x^{*}\right\|^{2}-2 \alpha_{n} \delta_{n}\left(1-\alpha_{n}\right)\left\langle x^{*}, x_{n}-x^{*}\right\rangle \\
= & \left(1-\alpha_{n} \delta_{n}\right) a_{n}+\delta_{n} \alpha_{n}\left(\alpha_{n}\left\|x^{*}\right\|^{2}+2\left(1-\alpha_{n}\right)\left\langle x^{*}, x^{*}-x_{n}\right\rangle\right) .
\end{aligned}
$$

Observing that

$$
\limsup _{n}\left[\alpha_{n}\left\|x^{*}\right\|^{2}-2\left(1-\alpha_{n}\right)\left\langle x^{*}, x^{*}-x_{n}\right\rangle\right] \leq 0,
$$

we can apply Lemma 2.5 to the relation (4.9) and conclude that $\lim _{n} a_{n}=0$; namely, $x_{n} \rightarrow x^{*}$ as required.

\section{ACKNOWLEDGMENTS}

The work of H.K. Xu was supported in part by NSC 97-2628-M-110-003-MY3. Part of this work was carried out while he was visiting the Tianjin Polytechnic University, sponsored in part by the Tianjin Municipal Higher Education Commission. He also extended his appreciation to the Deanship of Scientific Research at King Saud University for funding the work through the research group project No RGP-VPP-087.

\section{REFERENCES}

1. W. R. Mann, Mean value methods in iteration, Proc. Amer. Math. Soc., 4 (1953), 506510.

2. S. Reich, Weak convergence theorems for nonexpansive mappings in Banach spaces, $J$. Math. Anal. Appl., 67 (1979), 274-276.

3. A. Genel and J. Lindenstrauss, An example concerning fixed points, Isr. J. Math., 22 (1975), 81-86.

4. F. E. Browder and W. V. Petryshyn, Construction of fixed points of nonlinear mappings in Hilbert spaces, J. Math. Anal. Appl., 20 (1967), 197-228.

5. G. Marino and H. K. Xu, Weak and strong convergence theorems for strict pseudocontractions in Hilbert spaces, J. Math. Anal. Appl., 329(1) (2007), 336-346.

6. C. E. Chidume and S. A. Mutangadura, An example on the Mann iteration method for Lipschitz pseudo-contractions, Proc. Amer. Math. Soc., 129 (2001), 2359-2363. 
7. S. Ishikawa, Fixed points by a new iteration method, Proc. Amer. Math. Soc., 44 (1974), 147-150.

8. L. C. Ceng, A. Petrusel and J. C. Yao, Strong convergence of modified implicit iterative algorithms with perturbed mappings for continuous pseudocontractive mappings, Applied Math. Computation, 209 (2009), 162-176.

9. C. E. Chidume, M. Abbas and B. Ali, Convergence of the Mann iteration algorithm for a class of pseudocontractive mappings, Applied Math. Computation, 194 (2007), 1-6.

10. L. Ciric, A. Rafiq, N. Cakic and J. S. Ume, Implicit Mann fixed point iterations for pseudo-contractive mappings, Applied Math. Let., 22 (2009), 581-584.

11. K. Q. Lan and J. H. Wu, Convergence of approximants for demicontinuous pseudocontractive maps in Hilbert spaces, Nonlinear Anal., 49 (2002), 737-746.

12. C. Moore and B. V. C. Nnoli, Strong convergence of averaged approximants for Lipschitz pseudocontractive maps, J. Math. Anal. Appl., 260 (2001), 269-278.

13. X. Qin, Y. J. Cho, S. M. Kang and H. Zhou, Convergence theorems of common fixed points for a family of Lipschitz quasi-pseudocontractions, Nonlinear Anal., 71 (2009), 685-690.

14. Y. Yao, Y. C. Liou and G. Marino, A hybrid algorithm for pseudo-contractive mappings, Nonlinear Anal., 71 (2009), 997-5002.

15. Q. Zhang and C. Cheng, Strong convergence theorem for a family of Lipschitz pseudocontractive mappings in a Hilbert space, Math. Computer Modelling, 48 (2008), 480-485.

16. H. Zhou, Strong convergence of an explicit iterative algorithm for continuous pseudocontractions in Banach spaces, Nonlinear Anal., 70 (2009), 4039-4046.

17. S. Reich and A. J. Zaslavski, Convergence of Krasnoselskii-Mann iterations of nonexpansive operators, Math. Computer Modelling, 32 (2000), 1423-1431.

18. T. Suzuki, Strong convergence of approximated sequences for nonexpansive mappings in Banach spaces, Proc. Amer. Math. Soc., 135 (2007), 99-106

19. Y. Yao, Y. C. Liou and G. Marino, Strong convergence of two iterative algorithms for nonexpansive mappings in Hilbert spaces, Fixed Point Theory Appl., 2009 (2009), Article ID 279058, 7 pages, doi:10.1155/2009/279058.

20. X. Lu, H. K. Xu and X. Yin, Hybrid methods for a class of monotone variational inequalities, Nonlinear Anal., 71 (2009), 1032-1041.

21. H. K. Xu, Viscosity approximation methods for nonexpansive mappings, J. Math. Anal. Appl., 298 (2004), 279-291.

22. Y. Yao and J. C. Yao, On modified iterative method for nonexpansive mappings and monotone mappings, Applied Math. Computation, 186 (2007), 1551-1558.

23. K. Geobel and W. A. Kirk, Topics in Metric Fixed Point Theory, Cambridge Studies in Advanced Mathematics, Vol. 28, Cambridge University Press, 1990. 
24. K. Goebel and S. Reich, Uniform Convexity, Hyperbolic Geometry and Nonexpansive Mappings, Marcel Dekker, New York and Basel, 1984.

25. K. Deimling, Zero of accretive operators, Manuscripta Math., 13 (1974), 365-374.

26. H. K. Xu and R. G. Ori, An implicit iteration process for nonexpansive mappings, Numer. Funct. Anal. Optimiz., 22(5-6) (2001), 767-773.

27. R. H. Martin, Differential equations on closed subsets of Banach space, Trans. Amer. Math. Soc., 179 (1973), 399-414.

Yonghong Yao

Department of Mathematics

Tianjin Polytechnic University

Tianjin 300160

P. R. China

E-mail: yaoyonghong@yahoo.cn

Vittorio Colao and Giuseppe Marino

Dipartimento di Matematica

Universitá della Calabria

87036, Arcavacata di Rende (CS)

Italy

E-mail: colao@mat.unical.it gmarino@unical.it

Hong-Kun Xu

Department of Applied Mathematics

National Sun Yat-sen University

Kaohsiung 80424, Taiwan

and

Department of Mathematics

College of Science

King Saud University

P. O. Box 2455

Riyadh 11451

Saudi Arabia

E-mail: xuhk@math.nsysu.edu.tw 